\title{
FOTO-OXIDAÇÃO CATALÍTICA COM DIÓXIDO DE TITÂNIO APLICADA AO TRATAMENTO DE EFLUENTES TÊXTEIS EM ESCALA SEMI-PILOTO: AVALIAÇÃO DA REDUÇÃO DE COR, DA MATÉRIA ORGÂNICA E DA TOXICIDADE
}

\author{
D. C. LENHARD ${ }^{1}$, C. R. G. TAVARES ${ }^{2}$, A. Z. Dos SANTOS ${ }^{2}$, A. L. S. VOLPE ${ }^{2}$ \\ ${ }^{1}$ Universidade Tecnológica Federal do Paraná, Núcleo de Ciência de Alimentos \\ ${ }^{2}$ Universidade Estadual de Maringá, Departamento de Engenharia Química \\ E-mail para contato: daiane_lenhard@yahoo.com.br
}

\begin{abstract}
RESUMO - O trabalho teve como objetivo avaliar a redução da cor, da matéria orgânica (COT) e da toxicidade de um efluente têxtil industrial utilizando o processo de fotooxidação catalítica com $\mathrm{TiO}_{2}$. Os ensaios foram realizados em um reator batelada, utilizando-se $100 \mathrm{~L}$ de efluente com $\mathrm{pH}$ reduzido a 5, radiação UV fornecida por 42 lâmpadas de baixa pressão de $15 \mathrm{~W}$, dióxido titânio comercial e analítico em diferentes concentrações, e na presença de oxigênio (0,7 L.s-1). A redução de cor foi analisada pela redução do pico de absorbância em $660 \mathrm{~nm}$, enquanto as concentrações de Carbono Orgânico Total (COT) foram obtidas em aparelho Aurora 1030C TOC. Para a análise de toxicidade do efluente foi utilizado o microcrustáceo Artemia salina. Em todos os ensaios realizados houve redução da cor, do COT e da toxicidade do efluente, confirmando a eficiência do processo de foto-oxidação catalítica no tratamento do efluente têxtil.
\end{abstract}

\section{INTRODUÇÃO}

Os efluentes gerados nas indústrias têxteis apresentam grande diversidade e complexidade química originadas da grande variedade de fibras, corantes e produtos auxiliares utilizados pelas indústrias.

Além disso, estes efluentes caracterizam-se pela elevada concentração de cor, o que se deve à presença de corantes, e contém compostos orgânicos e inorgânicos que, em determinadas concentrações, podem ser tóxicos á vida aquática. A presença de corantes dissolvidos no efluente é bastante visível e, ao serem descartados sem tratamento, estes efluentes podem afetar a estética, a transparência, a solubilidade dos gases e os processos de fotossíntese nos corpos receptores, comprometendo a biota aquática.

Em função das implicações ambientais e de novas regulamentações quanto ao descarte de efluentes, novas tecnologias de tratamento avançado de efluentes vêm sendo pesquisadas (Duarte et al., 2013; Zheng et al., 2013; Baburaj et al., 2012; Faria et al., 2009; Rodrigues et al., 2009). Entre estas tecnologias, o processo de foto-oxidação catalítica tem se mostrado bastante promissor para o tratamento de corantes e efluentes têxteis, possibilitando inclusive a reutilização 
dos mesmos no processo de tingimento.

O processo é baseado na irradiação de um fotocatalisador, geralmente um semicondutor inorgânico, tal como $\mathrm{TiO}_{2}, \mathrm{ZnO}$, ou CdS, cuja energia do fóton deve ser maior ou igual a energia do "band gap" do semicondutor para provocar uma transição eletrônica. Assim, sob irradiação, um elétron é promovido da banda de valência para a banda de condução, gerando uma lacuna na banda de valência e elétrons na banda de condução (Ziolli e Jardim, 1998).

As lacunas mostram potenciais suficientemente positivos para gerar radicais hidroxilo, a partir de moléculas de água adsorvidas na superfície do semicondutor, os quais podem, conseqüentemente, oxidar contaminantes orgânicos (Nogueira e Jardim, 1998).

Recentemente, vários trabalhos foram publicados sobre a aplicação da foto-oxidação catalítica na degradação de corantes e efluentes têxteis (Rajamanickam e Shanthi, 2014; Sarwan et al., 2014; Chen et al., 2012; Gupta et al., 2012; Narayana et al., 2011; Satuf et al., 2011; Juang et al., 2010; Kuo e Lin, 2009; Marto et al., 2009; Jain e Shrivastava, 2008).

Este trabalho teve como principal objetivo investigar o tratamento do efluente gerado em uma lavanderia têxtil industrial pelo processo de foto-oxidação catalítica, a partir da avaliação da remoção de cor, do carbono orgânico total e da toxicidade.

\section{MATERIAIS E MÉTODOS}

\subsection{Materiais e reagentes}

Para os ensaios foi utilizado dióxido de titânio da Sigma-Aldrich, reagente analítico (BET $9,0 \mathrm{~m}^{2} \cdot \mathrm{g}^{-1}$ ), e também da Cosmo Chemical, reagente comercial (BET 7,2 $\mathrm{m}^{2} \cdot \mathrm{g}^{-1}$ ).

Os efluentes utilizados foram obtidos diretamente do tanque de armazenamento de uma lavanderia têxtil industrial, que tinge roupas prontas de jeans. $\mathrm{O}$ efluente consistia do efluente gerado nos processos da lavanderia, do dia corrente ou do dia anterior ao experimento.

\subsection{Procedimentos experimentais}

O reator, em escala semi-piloto, foi montado nas dependências de uma lavanderia industrial, para o tratamento do efluente da mesma. $O$ efluente foi alimentado ao reator por gravidade, e o volume a ser tratado, $100 \mathrm{~L}$, foi medido por um hidrômetro. O oxigênio foi fornecido ao reator por meio de um compressor de ar a uma vazão de $0,7 \mathrm{~L} \cdot \mathrm{s}^{-1}$. O pH do efluente foi ajustado para 5,0, utilizando-se ácido sulfúrico concentrado. Como fontes de radiação ultravioleta, foram utilizadas 42 lâmpadas a vapor de mercúrio de baixa pressão, de $15 \mathrm{~W}$ cada uma, fixadas em uma placa de aço inoxidável, encaixada sobre o reator. Uma borracha foi fixada na borda do reator para minimizar o impacto da chapa de inox e para evitar o vazamento de radiação ultravioleta para o exterior do tanque. 
Primeiramente foram realizados experimentos com os dois catalisadores na concentração de 1 g. $\mathrm{L}^{-1}$. Em seguida foram realizados ensaios somente com o $\mathrm{TiO}_{2}$ da Cosmo Chemical, nas concentrações de $0,5,1,0,1,5$ e 2,0 g.L-1.

Foram realizadas análises de Cor (Método HACH - Platina/Cobalto), DQO (Micro método), de COT (Medição direta no aparelho AURORA-TOC 1030C) e de descoloração no comprimento de onda de máxima absorbância.

Os testes de toxicidade foram realizados com o efluente antes e após o tratamento. A toxicidade foi avaliada utilizando-se o microcrustáceo Artemia salina, segundo metodologia adaptada de Meyer et al. (1982).

\section{RESULTADOS E DISCUSSÃO}

Os ensaios de foto-oxidação catalítica utilizando $\mathrm{TiO} 2$ como catalisador apresentaram significativas reduções na cor, DQO e COT dos efluentes analisados, conforme pode ser verificado pelos dados apresentados na Tabela 1.

Tabela 1 - Características dos efluentes submetidos ao tratamento por processo de foto-oxidação catalítica, utilizando $\mathrm{TiO}_{2}$.

\begin{tabular}{c|c|ccc}
\hline Catalisador & Parâmetros & $\begin{array}{c}\text { Cor } \\
(P t / C o\end{array}$ & $\begin{array}{c}\text { DQO } \\
\left(m g \mathrm{O}_{2} / \mathrm{L}\right)\end{array}$ & \multicolumn{1}{c}{ COT } \\
\hline \multirow{2}{*}{$\mathrm{TiO}_{2} /$ Sigma-Aldrich } & Antes do tratamento & 1388 & 305 & 38,92 \\
\cline { 2 - 5 }$\left(1,0\right.$ g.L $\left.{ }^{-1}\right)$ & Após o tratamento & 358 & 220 & 24,37 \\
\cline { 2 - 5 } & Redução & $74,2 \%$ & $27,9 \%$ & $37,4 \%$ \\
\hline $\mathrm{TiO}_{2} /$ Cosmo Chemical & Antes do tratamento & 994 & 210 & 44,78 \\
\cline { 2 - 5 }$\left(1,0\right.$ g.L $\left.{ }^{-1}\right)$ & Após o tratamento & 192 & 137 & 23,93 \\
\cline { 2 - 5 } & Redução & $80,7 \%$ & $34,8 \%$ & $46,6 \%$ \\
\hline
\end{tabular}

A varredura espectrofotométrica do efluente submetido ao tratamento por foto-oxidação catalítica, utilizando $\mathrm{TiO}_{2}$ da Sigma-Aldrich, apresentou pico máximo de absorbância em $660 \mathrm{~nm}$ e a descoloração do efluente, após 240 minutos, neste comprimento de onda, foi de $60 \%$. Já o efluente submetido ao tratamento com $\mathrm{TiO}_{2}$ da Cosmo Chemical apresentou pico máximo de absorbância em 600nm, e a descoloração no pico, após 240 minutos, foi de 66\%. Os experimentos posteriores foram realizados com $\mathrm{TiO}_{2}$ da Cosmo Chemical, pois a utilização deste representa um menor custo no tratamento, com resultados tão satisfatórios quanto os obtidos utilizando-se o catalisador analítico (Sigma-Aldrich).

Ao se variar a concentração de $\mathrm{TiO}_{2}$ nos ensaios de tratamento dos efluentes têxteis verificou-se que a maior porcentagem de descoloração foi obtida utilizando-se 0,5 g.L $\mathrm{L}^{-1}$ de $\mathrm{TiO}_{2}$ conforme apresentado na Figura 1. Este resultado pode ser justificado pelo fato de uma menor concentração de catalisador permite a maior penetração dos raios ultravioleta, facilitando a absorção pelas moléculas e favorecendo o processo de fotodegradação. A utilização de menores 
concentrações de catalisador é um fator positivo, pois resulta em menor geração de resíduo final e menor custo.

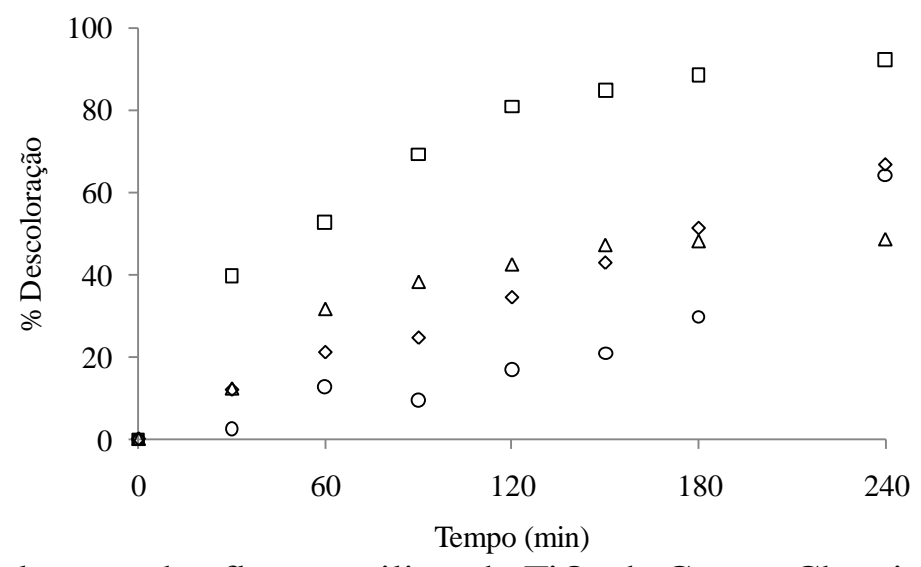

Figura 1 - Descoloração do efluente utilizando $\mathrm{TiO}_{2}$ da Cosmo Chemical em diferentes concentrações: $(\diamond)-1,0$ g.L ${ }^{-1} ;(\square)-0,5$ g.L $L^{-1} ;(\Delta)-1,5$ g.L ${ }^{-1} ;(\circ)-2,0$ g.L ${ }^{-1}$.

No entanto, novos testes foram realizados nas concentrações de 1,0 e 0,5 g.L $\mathrm{L}^{-1}$ de $\mathrm{TiO}_{2}$, para confirmação dos resultados obtidos. Nestes novos testes, verificou-se uma média de $90,1( \pm$ $6,7) \%$ na descoloração dos efluentes quando utilizado o catalisador na concentração maior e uma média de 65,3 ( \pm 19,7)\% de descoloração ao se utilizar o catalisador em menor concentração (Tabela 2). A utilização de maior concentração de catalisador, além de proporcionar maior média de descoloração também apresentou menor desvio entre os resultados. O desvio verificado foi inferior a $10 \%$ indicando boa reprodutibilidade dos experimentos, considerando-se que foram realizados com efluentes coletados em dias diferentes.

Além disso, o ajuste cinético dos dados, apresentado na Figura 2 e Tabela 2, mostrou que a reação segue cinética de primeira ordem, sendo que as maiores velocidades de descoloração também foram verificadas nos experimentos com 1,0 g. $\mathrm{L}^{-1}$ de $\mathrm{TiO}_{2}$.
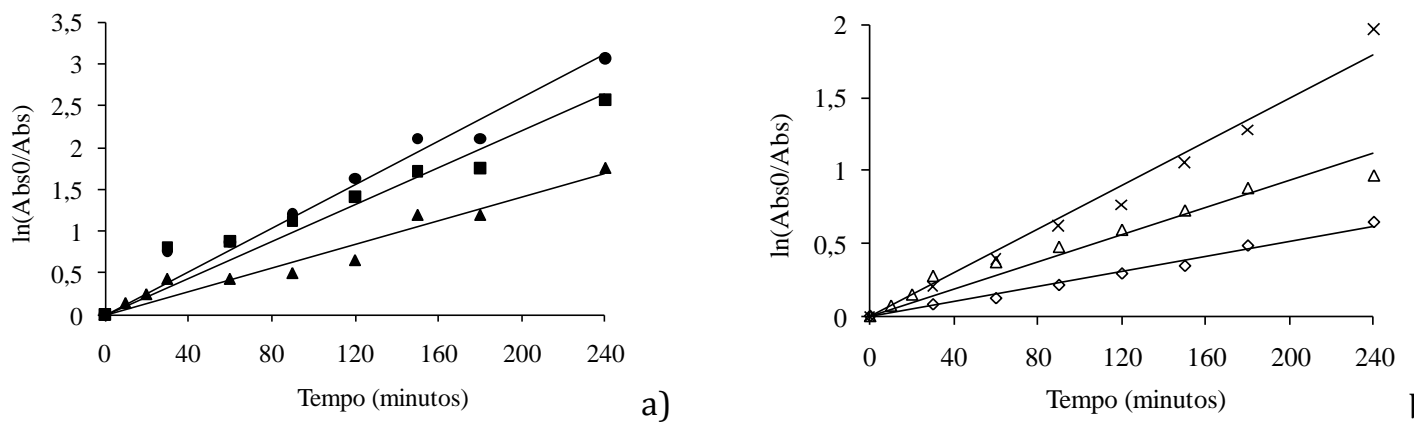

Figura 2 - Ajustes cinéticos de descoloração dos efluentes utilizando $\mathrm{TiO}_{2}$ nas concentrações de:

(a) 1,0 g.L ${ }^{-1}$, $\bullet$ exp. 1, $\square$ exp. 2, $\mathbf{\Delta} \exp .3$; (b) 0,5 g.L ${ }^{-1}, \diamond \exp .4$, x exp. $5, \Delta$ exp. 6. 
Tabela 2 - Parâmetros cinéticos da descoloração de efluentes têxteis utilizando $\mathrm{TiO}_{2}$ comercial nas concentrações de 1,0 g. $\mathrm{L}^{-1}$ e $0,5 \mathrm{~g} . \mathrm{L}^{-1}$.

\begin{tabular}{ccccc}
\hline & Experimento & $\begin{array}{c}\text { \% Descoloração } \\
\text { Após 240 minutos }\end{array}$ & $K\left(\mathrm{~min}^{-1}\right)$ & $\begin{array}{c}\text { Coeficiente de } \\
\text { correlação }\end{array}$ \\
\hline \multirow{3}{*}{1,0 g.L ${ }^{-1}$} & 1 & 95,33 & 0,013 & $\mathrm{R}^{2}=0,964$ \\
& 2 & 92,36 & 0,011 & $\mathrm{R}^{2}=0,912$ \\
& 3 & 82,61 & 0,007 & $\mathrm{R}^{2}=0,947$ \\
\hline 0,5 g.L & 4 & 47,69 & 0,002 & $\mathrm{R}^{2}=0,985$ \\
& 5 & 86,05 & 0,007 & $\mathrm{R}^{2}=0,977$ \\
& 6 & 62,07 & 0,004 & $\mathrm{R}^{2}=0,941$ \\
\hline
\end{tabular}

A influência da concentração de catalisador na cinética de descoloração de duas soluções de corantes usando concentrações de $\mathrm{TiO}_{2}$ variando de 0,25 até 4,0 g.L $\mathrm{L}^{-1}$ também foi investigada por Bizani et al. (2006). Os autores observaram que a taxa de descoloração aumenta com o aumento da concentração do catalisador até o limite de 1,0 g. $\mathrm{L}^{-1}$, a partir do qual nenhuma melhora é observada. Os autores afirmam que o platô, observado quando certa quantidade de catalisador é adicionada, pode ser uma consequiência do fato da reação ocorrer nas moléculas de corante adsorvidas na superfície do catalisador. Então, quando todas as moléculas de corante estão adsorvidas na superfície do $\mathrm{TiO}_{2}$, nenhuma melhora é obtida adicionando-se mais catalisador. A diminuição na eficiência, que foi observada para os dois corantes, com o aumento da quantidade de catalisador, pode ser atribuída, segundo os autores, a um aumento da opacidade da suspensão e a um aumento da refletância da luz, devido ao excesso de partículas de $\mathrm{TiO}_{2}$.

A mineralização parcial dos efluentes pode ser verificada pela redução do carbono orgânico total dos efluentes, após 240 minutos. Nos experimentos com 1,0 g.L $\mathrm{L}^{-1}$ de $\mathrm{TiO}_{2}$ houve uma redução média de 33,9 ( \pm 6,8) \% no COT, enquanto nos experimentos com 0,5 g.L-1 de TiO2 a redução média do COT foi de $23,7( \pm 8,8) \%$.

A redução do COT também seguiu cinética de primeira ordem, sendo que as maiores velocidades de reação mais uma vez foram verificadas nos experimentos com maior concentração de $\mathrm{TiO}_{2}$, conforme apresentado na Figura 6 e na Tabela 3.

Verifica-se que não houve mineralização completa dos efluentes, uma vez que não ocorreu redução total do carbono orgânico, portanto o efluente final contém produtos desta reação, ou seja, compostos gerados na quebra dos grupos cromóforos. Como não foi possível identificar estes compostos, foram realizados testes para se comparar a toxicidade do efluente antes e após o tratamento foto-oxidativo. 


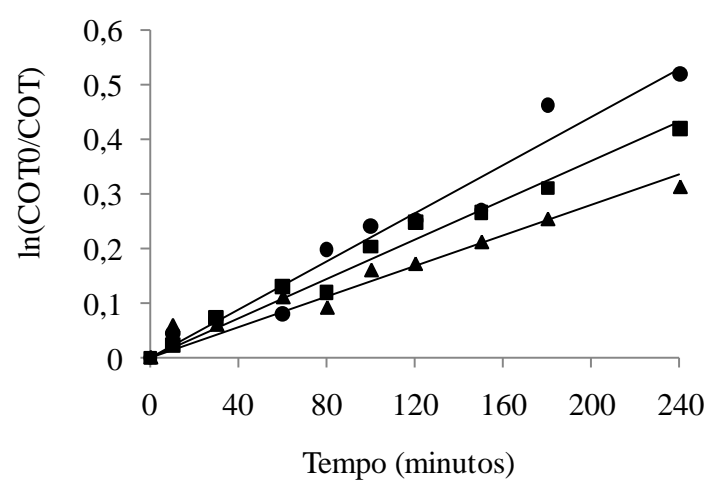

a)

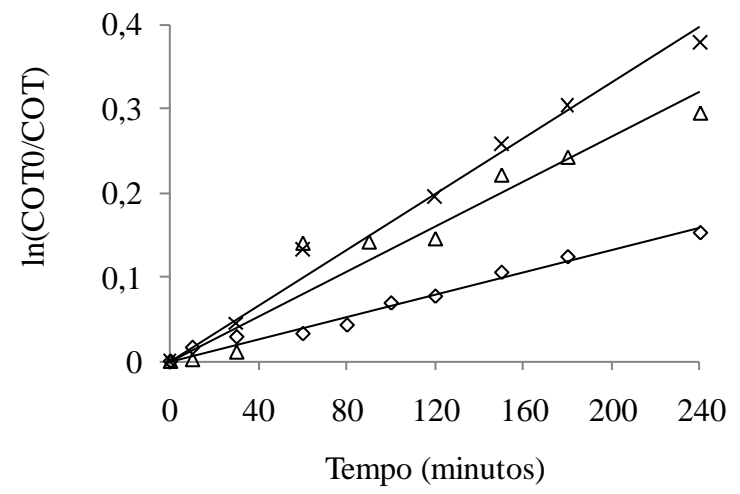

b)

Figura 6. Ajustes cinéticos de redução de COT dos efluentes utilizando $\mathrm{TiO}_{2}$ comercial nas

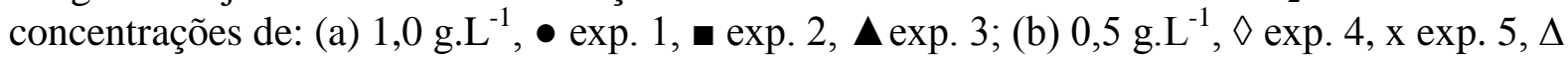
exp. 6.

Tabela 3. Parâmetros cinéticos de redução de COT de efluentes têxteis utilizando $\mathrm{TiO}_{2}$ comercial nas concentrações de 1,0 g.L $\mathrm{L}^{-1}$ e 0,5 g.L. ${ }^{-1}$.

\begin{tabular}{ccccc}
\hline & Experimento & $\begin{array}{c}\text { \% Redução de COT } \\
\text { Após 240 minutos }\end{array}$ & $K\left(\mathrm{~min}^{-1}\right)$ & $\begin{array}{c}\text { Coeficiente de } \\
\text { correlação }\end{array}$ \\
\hline \multirow{3}{*}{$1,0 \mathrm{~g} . \mathrm{L}^{-1}$} & 1 & 40,53 & 0,0022 & $\mathrm{R}^{2}=0,954$ \\
& 2 & 34,21 & 0,0018 & $\mathrm{R}^{2}=0,979$ \\
& 3 & 26,93 & 0,0014 & $\mathrm{R}^{2}=0,948$ \\
\hline \multirow{2}{*}{0,5 g.L ${ }^{-1}$} & 4 & 14,14 & 0,0007 & $\mathrm{R}^{2}=0,980$ \\
& 5 & 31,54 & 0,0017 & $\mathrm{R}^{2}=0,986$ \\
& 6 & 25,54 & 0,0013 & $\mathrm{R}^{2}=0,934$ \\
\hline
\end{tabular}

Os resultados dos testes de toxicidade realizados para os efluentes gerados nos ensaios com 1,0 e 0,5 g. $\mathrm{L}^{-1}$ de catalisador demonstraram redução na taxa de mortalidade da Artemia salina no efluente após o tratamento por foto-oxidação catalítica, como pode ser verificado na Tabela 4.

Tabela 4 - Taxas de mortalidade observadas pelo teste de toxicidade com Artemia salina, nos experimentos de tratamento de efluentes têxteis pelo processo foto-oxidativo com diferentes concentrações de $\mathrm{TiO}_{2}$.

\begin{tabular}{ccc}
\hline & \multicolumn{2}{c}{ Taxa de mortalidade (\%) } \\
\cline { 2 - 3 } EXPERIMENTO & $\begin{array}{c}\text { Efluente sem } \\
\text { tratamento }\end{array}$ & $\begin{array}{c}\text { Tratado por foto- } \\
\text { oxidação catalítica }\end{array}$ \\
\hline 1 & 36,84 & 15,29 \\
2 & 10,53 & 00,00 \\
3 & 43,48 & 11,76 \\
\hline 4 & 30,00 & 6,25 \\
5 & 31,58 & 10,53 \\
6 & 43,48 & 21,05 \\
\hline
\end{tabular}




\section{CONCLUSÕES}

O processo de foto-oxidação catalítica, mostrou-se eficiente no tratamento de efluentes de lavanderias têxteis, no que diz respeito à descoloração/degradação dos mesmos, além de contribuir para a redução da toxicidade destes efluentes.

Apesar dos efluentes utilizados terem sido coletados em dias diferentes, de forma geral, as maiores descolorações foram obtidas utilizando-se dióxido de titânio na concentração de 1 g.L. ${ }^{-1}$. No entanto, bons resultados também foram obtidos utilizando-se o catalisador na concentração de 0,5 g. $\mathrm{L}^{-1}$.

\section{AGRADECIMENTOS}

Os autores agradecem à Fundação Araucária, ao SEBRAE, à FINEP, à Universidade Tecnológica Federal do Paraná e à Universidade Estadual de Maringá pelo apoio.

\section{REFERÊNCIAS}

BABURAJ, M.S.; ARAVINDAKUMAR, C.T.; SREEDHANYA, S.; THOMAS, A.P. e ARAVIND, U. K. Treatment of model textile effluents with PAA/CHI and PAA/PEI composite mebranes, Desalin. v. 288, p. 72-79, 2012.

SARWAN, B.; PARE, B. e ACHARYA, A.D. Heterogeneous photocatalytic degradation of nile blue dye in aqueous BiOCl suspensions, App. Surf. Sci. v. 301, p. 99-106, 2014.

BIZANI, E.; FYTIANOS, K.; POULIOS, I. e TSIRIDIS, V. Photocatalytic decolorization and degradation of dye solutions and wastewaters in the presence of titanium dioxide. J. Hazard. Mater. v. 136, p. 85-94, 2006.

DUARTE, F.; MALDONADO-HÓDAR, F.J.; MORAIS,V. e MADEIRA, L.M. Treatment of textile effluents by the heterogeneous Fenton process in a continuous packed-bed reactor using Fe/activated carbon as catalyst. Chem. Eng. J. v. 232, p. 34-41, 2013.

Faria, P.C.C., Órfão, J.J.M., Pereira, M.F.R., Activated carbon and ceria catalysts applied to the catalytic ozonation of dyes and textile effluents. App. Catal. B: Environ. 88 (2009) 341-350.

CHEN X.; WANG W.; XIAO H.; HONG C.; ZHU F.; YAO Y. e XUE Z. Accelerated TiO 2 photocatalytic degradation of Acid Orange 7 under visible light mediated by peroxymonosulfate, Chem. Eng. J. v. 193-194, p. 290-295, 2012.

GUPTA V. K.; JAIN R.; MITTAL A.; SALEH T.A.; NAYAK A.; AGARWAL S. E SIKARWAR S. Photo-catalytic degradation of toxic dye amaranth on $\mathrm{TiO}_{2} / \mathrm{UV}$ in aqueous suspension, Mater. Sci. and Eng.: C. v.32, p. 12-17, 2012.

JAIN R. e SHRIVASTAVA M. Photocatalytic removal of hazardous dye cyanosine from industrial waste using titanium dioxide, J. Hazard. Mater. v. 152, p. 216-220, 2008.

JUANG R.; LIN S. e HSUEH P. Removal of binary azo dyes from water by UV-irradiated degradation in TiO2 suspensions, J. Hazard. Mater. v. 182, p. 820-826, 2010. 
KUO C. e LIN H. Photodegration of C.I. Reactive Red 2 by platinized titanium dioxide, J. Hazard. Mater. v. 165, p. 1243-1247, 2009.

MARTO J.; MARCOS P. S.; TRINDADE T. e LABRINCHA J.A. Photocatalytic decolouration of Orange II by $\mathrm{ZnO}$ active layers screen-printed on ceramic tiles, J. Hazard. Mater. v. 163, p. 3642, 2009.

MEYER, B. N.; FERRIGNI, N. R.; PUTNAM, J. E.; JACOBSEN, L. B.; NICHOLS, D. E. e McLAUGHLIN, J. L. Brine Shrimp: A convenient gereral bioassay for active plant constituents. J. Medic. Plant Res, v. 45, p. 31-34, 1982.

NARAYANA R. L.; MATHESWARAN M.; AZIZA. A. e P. SARAVANAN, Photocatalytic decolourization of basic green dye by pure and $\mathrm{Fe}$, Co doped $\mathrm{TiO} 2$ under daylight illumination, Desalination. v. 269, p. 249-253, 2011.

NOGUEIRA, R. F. P. e JARDIM, W. F. A fotocatálise heterogênea e sua aplicação ambiental. Quím. N. v. 21, p. 69-72, 1998.

RAJAMANICKAM, D. e SHANTHI, M. Photocatalytic degradation of an azo dye Sunset Yellow under UV-A light using $\mathrm{TiO}_{2} / \mathrm{CAC}$ composite catalysts. Spectroc. Ac. Part A: Molec. Biomol. Spect. v. 128, p. 100-108, 2014.

RODRIGUES, C. S. D.; MADEIRA, L. M. d BOAVENTURA, R. A. R. Treatment of textile effluent by chemical (Fenton's Reagent) and biological (sequencing batch reactor) oxidation. J. Hazard. Mater. v. 172, p. 1551-1559, 2009.

SATUF M. L.; PIERRESTEGUI M. J.; ROSSINI L.; BRANDI R. J. e ALFANO O. M., Kinetic modeling of azo dyes photocatalytic degradation in aqueous $\mathrm{TiO}_{2}$ suspensions. Toxicity and biodegradability evaluation, Catal. Today. v. 161, p. 121-126, 2011.

ZHENG, Y.; YU, S.; SHUAI, S.; ZHOU, Q.; CHENG, Q.; LIU, M. e GAO, C. Color removal and COD reduction of biologically treated textile effluent through submerged filtration using hollow fiber nanofiltration membrane. Desalin. v. 314, p. 89-95, 2013.

ZIOLLI, R.L. e JARDIM, W.F., Mecanismo de fotodegradação de compostos orgânicos, catalisada por TiO2. Quím. N. v. 21, p. 319-325, 1998. 